\title{
Appropriate Use and Future Directions of Molecular Diagnostic Testing
}

\author{
Erin H. Graf ${ }^{1}$ • Preeti Pancholi ${ }^{2}$ \\ Published online: 6 February 2020 \\ (C) Springer Science+Business Media, LLC, part of Springer Nature 2020
}

\begin{abstract}
Purpose of Review Major technologic advances in two main areas of molecular infectious disease diagnostics have resulted in accelerated adoption or ordering, outpacing implementation, and clinical utility studies. Physicians must understand the limitations to and appropriate utilization of these technologies in order to provide cost-effective and well-informed care for their patients.

Recent Findings Rapid molecular testing and, to a lesser degree, clinical metagenomics are now being routinely used in clinical practice. While these tests allow for a breadth of interrogation not possible with conventional microbiology, they pose new challenges for diagnostic and antimicrobial stewardship programs. This review will summarize the most recent literature on these two categories of technologic advances and discuss the few studies that have looked at utilization and stewardship approaches. This review also highlights the future directions for both of these technologies.

Summary The appropriate utilization of rapid molecular testing and clinical metagenomics has not been well established. More studies are needed to assess their prospective impacts on patient management and antimicrobial stewardship efforts as the future state of infectious disease diagnostics will see continued expansion of these technologic advances.
\end{abstract}

Keywords Syndromic panels · Multiplex molecular panels · CLIA-waived molecular tests · Clinical metagenomics · Next-generation sequencing $\cdot$ Diagnostic stewardship

\section{Introduction}

While the first direct-specimen molecular testing for infectious diseases was approved by the US FDA 26 years ago [1], there has been a very recent explosion of molecular technology in two major directions: (1) direct-specimen rapid amplification and detection platforms and (2) next-generation sequencing. These advanced techniques offer an opportunity to provide laboratory diagnoses at a speed, sensitivity, and

This article is part of the Topical Collection on Technology and Infectious Disease

Preeti Pancholi

Preeti.Pancholi@osumc.edu

Erin H. Graf

Graf.Erin@mayo.edu

1 Department of Laboratory Medicine and Pathology, Mayo Clinic Arizona, Phoenix, AZ, USA

2 Department of Pathology, The Ohio State University Wexner Medical Center, Columbus, OH, USA breadth never before possible with conventional microbiology. Given the continued emergence and spread of novel antimicrobial resistance mechanisms paired with increasingly complex patient populations, these technologic advances may become important factors in patient care. However, each comes with its own set of significant limitations including cost considerations and potentially negative consequences, such as over-utilization. As a result, there is also room for novel stewardship strategies around these types of testing.

One of the early applications in this wave of technology is species and antimicrobial resistance marker identification from positive blood culture broth. Several rapid, multiplexed molecular panels received US FDA clearance in the last 5 to 10 years [2]. These platforms provide significantly decreased turnaround times for limited organism and resistance marker identification. However, numerous studies, some almost a decade old, have shown that without any intervention, simply implementing this relatively expensive technology has no clinical or economic impact [3-5]. What we have learned from these studies is that in order to see some benefit from this specific technology, an antimicrobial stewardship 
program (ASP) must be engaged to direct changes in treatment. Only through this approach can the technology lead to faster time to optimal therapy, shorter hospital stays, and associated hospital cost savings for very specific scenarios [3-5]. Although this is not a direct-specimen application, we can begin to draw parallels with direct-specimen molecular testing. This review will highlight recent studies developing and applying emerging molecular infectious disease technologies and touch on limited published data on clinical utility and stewardship approaches.

\section{Rapid Molecular Testing}

\section{Syndromic Panels}

"Syndromic panel" is a term commonly used in clinical microbiology when referring to rapid, multiplexed amplification and detection platforms that received US FDA approval or clearance for a set list of targets in a specific sample type [6]. This includes panels for gastrointestinal, central nervous system and respiratory tract infection-associated potential pathogens. There are common themes among all of these panels. First, they are superior in analytical sensitivity and speed compared to the syndromic approaches they replaced, that is viral and bacterial cultures. Second, due to the fact that they emerged and were adopted quickly as a superior method to culture, adoption has outpaced evidence of clinical utility for some tests and populations [7]. Ideally, we would evaluate all of these approaches via randomized controlled trials comparing patient outcomes between conventional microbiologic testing and testing including a syndromic panel; however, very limited studies addressing this have been published [8]. Finally, there are also very limited studies on appropriate utilization. Given the increased cost over conventional methods and potential unintended consequences of over-testing, these are areas in need of exploration [9]. How these points relate to each panel type will be discussed separately below.

\section{Respiratory Tract Infection Syndromic Panels}

For a detailed review of individual respiratory tract rapid syndromic respiratory panels, readers are referred to the following references $(6,8)$. While individual targets vary, in general, these panels contain most known, widely circulating respiratory viruses and a limited set of atypical upper respiratory tract bacterial targets. Analytical sensitivity also varies by target for each panel. A number of studies have attempted to evaluate whether implementation of these panels results in any impact on patient care. Several retrospective studies have shown mixed results when considering impact on antibiotic usage, including duration, or length of hospitalization when comparing pre- and post-panel implementation time periods or comparing patients with positive versus negative panel results [10-14]. One of the only randomized controlled trials also found no difference in antibiotic usage, including duration, between adults tested via syndromic panel at the point-of-care compared to patients receiving conventional testing in a hospital emergency department and inpatient unit [15]. They attributed this to the fact that antibiotics were initiated before the syndromic panel was completed. Upon secondary analysis, only including patients who were not prescribed antibiotics prior to receiving the panel result, they found a small $(13 \%)$ but statistically significant difference in antibiotic prescriptions. The same study also found that patients in the syndromic panel arm were discharged a mean of 1 day earlier than those receiving conventional testing. Another randomized controlled trial found no difference in length of stay or antibiotic usage, including duration, comparing adult patients who received syndromic panel testing with those who received conventional microbiologic testing [16]. In many of these studies, there appears to be a significant impact only when patients test positive for influenza virus. For example, in a retrospective pre-, postimplementation analysis, Rappo et al. found that an influenza diagnosis by rapid syndromic panel, compared to conventional testing, led to less admissions, shorter length of stay and duration of antibiotics, as well as less imaging [11]. In the same study, there was no impact when patients were positive for a noninfluenza virus by syndromic panel compared to conventional testing. This leaves an obvious place for antimicrobial and diagnostic stewardship. ASP could provide practice-level guidance on the proper interpretation of these results; that is, with-hold antibiotics if a virus is detected when other clinical features or testing is not consistent with bacterial infection [17]. Trials evaluating the success of these types of interventions are needed.

The future of respiratory syndromic panels includes lower respiratory tract sampling. Two panels have recently been cleared by the US FDA for sputum, endotracheal aspirate and bronchoalveolar lavage specimens. One of these panels includes the same viral targets as their upper respiratory tract panel, while both have the same 18 bacterial targets and 8 to 10 antibiotic resistance genes [6]. This expansive number of bacterial targets may lead to tremendous confusion for the ordering physician if many targets are positive on the same sample, including antibiotic resistance genes that can be attributed to multiple species. One manufacturer provides semiquantitative assessment, via 10-log increments, of most of the bacterial targets to attempt to aid in interpretation. However, it is not clear what relative quantities should be considered significant. At the time of this writing, there are no published studies available; thus, we cannot comment on the clinical performance or appropriate utility of such panels.

\section{Gastrointestinal Tract Infection Syndromic Panels}

As with respiratory panels, there are a number of US FDAapproved rapid syndromic panels for gastroenteritis reviewed in the following references $(6,8)$. While these tests have 
improved sensitivity and reduced time to result over bacterial culture for common agents of community-acquired diarrhea, they introduce new issues. First, one of the panels contains an expansive list of potential pathogens, including several low incidence targets and four $E$. coli targets, only one of which is tested for as part of a standard stool culture. In early clinical studies, Enteropathogenic E. coli (EPEC) was the most frequently identified organism on the panel across all age groups $[18,19]$. While this organism is a cause of pediatric diarrhea in developing countries, the unexpected prevalence in adults and a population without travel to endemic areas raised concerns about detection of colonization. EPEC was also more frequently found as part of co-detections than alone. Further studies have repeated these findings in countries across different continents and have also identified the same issues with enteroaggregative $E$. coli [20-22]. False-positive detections of Vibrio cholerae [23] and Entamoeba histolytica [24] have been reported in patients without relevant exposures. This highlights the inherent problems with testing patients for potential pathogens without clinical indication, especially in low prevalence settings. To combat potential negative consequences of broad panel testing, antimicrobial and diagnostic stewardship partnerships are critical [25]. To this point, Keske et al. evaluated the inappropriate use of antibiotics, defined as the use of antibiotics after a pathogen was detected that did not require treatment with that drug, during a pre- and post-ASP intervention at a single hospital in Turkey. The ASP intervention was described as "educational meetings and activities among prescribers" that took place at the end of the preintervention time period. The authors saw a significant reduction in inappropriate prescriptions even with this modest intervention [22]. Importantly, analysis was limited to adults with severe diarrhea and fever or bloody diarrhea. The authors did not state whether testing was also limited to this population as a diagnostic stewardship approach. To evaluate inappropriate utilization, Clark et al. performed retrospective chart review on all adult outpatients from the University of Virginia health system tested by syndromic GI panel in a 15-month period. They found that $32 \%$ of patients were tested inappropriately, that is, not meeting the Infectious Disease Society of America's recommendations [26]. Of these $32 \%$ of patients deemed inappropriately tested, only one had a clinically significant pathogen detected but this detection was deemed not to have impacted management.

Perhaps the most concerning impact of implementing a syndromic GI panel without diagnostic stewardship is the potential over-diagnosis of $C$. difficile infection (CDI) in colonized individuals and related financial consequences [27]. The IDSA currently recommends limiting testing to individuals greater than 2 years of age with diarrhea following antimicrobial use and/or healthcare-acquired diarrhea, though individuals with persistent diarrhea lacking an etiology may be considered for testing [26]. These categories are complicated by the association of many medications, including antimicrobials, with diarrhea, as well as overlapping symptoms between CDI and viral causes of hospital-acquired diarrhea and increased likelihood of $C$. difficile colonization in individuals with antibiotic and healthcare exposures [28]. There is intense debate around the use of antigen testing versus molecular for the diagnosis of CDI, which is beyond the scope of this review [29]. Suffice to say that diagnostic and ASP partnerships are necessary to reduce syndromic GI panel testing that detects colonization and subsequent unnecessary treatment. In a prospective study, Truong et al. performed real-time enforcement of testing criteria to reject specimens for $C$. difficile molecular testing that were from patients without diarrhea or who had received laxatives. Through this intervention, they were able to detect significant reductions in tests ordered, use of oral vancomycin, and rates of hospital onset CDI [30]. While the test used in this study was not a syndromic panel, the same conclusions can be drawn to increase the value of GI panel testing.

\section{Central Nervous System Infection Syndromic Panels}

At the time of this writing, there is only one US FDAapproved multiplex molecular panel for the detection of central nervous system infection-associated pathogens in cerebrospinal fluid collected through lumbar puncture [31]. However, it is a matter of when, not if, more will be cleared [32]. We are now roughly 4 years out from the initial approval of the first panel with many lessons learned. The clinical trial and several retrospective studies performed around the time of release revealed some concerning findings [31, 33]. First, falsepositive results for bacteria found in the respiratory tract and herpes simplex virus (HSV) were commonly reported [31, 33]. The origin of this false-positivity has been hypothesized to be contamination from medical laboratory scientists, individuals collecting samples or other clinical samples processed in the same location; however, this has not been scientifically proven. Second, Cryptococcal meningitis should still be diagnosed by antigen testing due to superior sensitivity of that method [33-35]. Third, detection of HHV-6 is common and of questionable clinical significance [36-38]. Laboratories should provide an interpretive comment on the report for HHV-6 detections possibly prompting consultation with infectious diseases, though this leads to additional consult burden on these clinical teams. Finally, some targets were not adequately represented in the clinical trial due to infrequent clinical presentations, which could have been predicted by statistical estimates. As a result, the sensitivity of detection for bacterial meningitis is not well documented and there is a published example of the panel failing to detect Neisseria meningitidis in a sample that was only positive after a 5-fold dilution, which is a deviation from the FDA approval [39]. The authors hypothesized that the patient's CSF's high white 
blood cell count could have led to interference. More likely, the organism DNA load was so high that it diluted speciesspecific primers and probes. Since the panel also does not detect all possible causes of bacterial meningitis culture is still required. Additionally, the panel has been shown to have lower analytical sensitivity for HSV than single-target molecular tests [40, 41]. Thus, negative panel results in the setting of high clinical suspicion for HSV encephalitis or neonatal disseminated disease should be interpreted with caution.

The speed and ease of testing has led to the rapid adoption of this platform in many laboratories before clinical performance and utility could be fully established. For example, many labs chose to adopt due to the fact that send out testing for HSV was expensive and led to delays [43]. The syndromic panel allows them to perform testing in house at perhaps the same cost with the potential benefit of the additional targets. However, as mentioned above, the lower analytical sensitivity for HSV raises concern. Some argue that this new ease of testing has possibly led to overuse $[42,43]$. However, retrospective analyses have the benefit of knowing the final diagnosis while at the time of clinical presentation, it may have been appropriate to order syndromic panel testing [43]. Studies that have looked at pre- and post-panel implementation time-points have found conflicting differences in panel impact in terms of length of stay and time to appropriate antimicrobial narrowing, with some showing significant decreases $[44,45]$ and others showing no difference [46]. Since there is no set guidance for how or if testing should be limited as a stewardship approach, it is currently unclear how to gain the most benefit from this panel. Some have proposed a CSF cell count-based cutoff to limit testing [47]. Such cutoffs are clearly not universal to all populations or infections [48]. For example, HSV encephalitis can often present with a normal CSF cell count early in the course of disease [49].

\section{Reimbursement Concerns with Syndromic Panels}

The future for syndromic panel testing may require smaller, modular, or even reflexed panels to achieve maximum financial and clinical benefit. This is in part due to recent Centers for Medicare and Medicaid Services' (CMS) local coverage determinations (LCD) that deemed some components of these panels not reasonable or necessary. LCDs limit reimbursement to five bacterial targets on the syndromic GI panel, unless C. difficile colitis is suspected and tested as a part of the panel, and three to five viral targets on the respiratory syndromic panels (CMS, https://www.cms.gov/medicare-coveragedatabase/details/lcd-details. aspx ?LCDId $=37363$ \&ver $=3$, accessed 10/30/2019). To potentially combat this, some manufacturers have received proprietary laboratory analyses (PLA) codes (American Medical Association, https://www. ama-assn.org/system/files/2019-10/cpt-pla-codes-long.pdf, accessed 10/30/2019), which in theory allow for exemption from the LCDs. However, at the time of this writing, it is unclear if all payers will accept these new codes and what the reimbursement rate will be. Studies addressing the downstream financial benefits of testing, such as savings from reduced antibiotic use or decreased length of hospitalizations, and how these offset the cost of the testing are needed [50].

\section{Point-of-Care Molecular}

Without question, the future of rapid, random-access molecular testing is moving closer and closer to the bedside or clinic room. Currently, there are a number of Clinical Laboratory Improvement Amendments (CLIA)-waived molecular tests for Influenza virus, some paired with respiratory syncytial virus, group A Streptococcal pharyngitis and for Chlamydia trachomatis and Neisseria gonorrhoeae (CT/NG) with excellent analytical sensitivity and specificity $[51,52]$. CLIAwaived means that these tests can be performed outside of a laboratory and by non-laboratory staff such as nurses. Studies of these platforms have shown clinical impact for influenza detection in both the inpatient and ambulatory settings [53, 54]. In a prospective, single-season study of urgent care centers connected to their health system, Benirschke et al. found a significant reduction in the use of antibiotics for those who tested positive for influenza by a POC molecular test compared to those who tested positive by a POC antigen test [54]. Martinot et al. had similar significant reductions in length of stay, antibiotic administration, and faster oseltamivir administration after a positive influenza test in an emergency department population comparing POC molecular to a slower laboratory developed molecular influenza test [55]. Group A Streptococcal (GAS) pharyngitis POC molecular tests are now CLIA-waved without the need for confirmatory culture of negative results. In a prospective primary care study comparing a CLIA-waved POC molecular test to conventional methods for GAS, Rao et al. found a small but significant reduction in inappropriate antibiotic use for children presenting with symptoms of GAS pharyngitis [56]. For CT/NG, the majority of these tests are currently sent to central laboratories, leaving up to a 7-day waiting period from first visit to treatment. During that time, patients may spread infection, and many patients receiving positive results do not return for treatment. These POC tests allow people to be tested, diagnosed, and treated for STIs in a single visit $[57,58]$. They can also help to reduce the burden on health workers and systems and can therefore also reduce expensive laboratory costs.

Bringing molecular testing outside of the clinical laboratory comes with appropriate concern. First, these assays produce millions to trillions of copies of pathogen nucleic acid. While they are closed systems, there is always the possibility of amplicon contamination from a defective product and one laboratory accrediting agency has recently added new 
requirements to this point [59]. To investigate potential contamination, Donato et al. performed weekly swabs of the surface of an actively used POC molecular GAS test and surrounding environment at two different urgent care clinics [60]. They were not able to detect any contamination that amplified via the same test across the 13-week period of the study. Second, as more molecular assays become CLIAwaved for POC use, especially those with multiple targets, the ability to provide diagnostic stewardship becomes increasingly challenging. For example, one of the large syndromic respiratory panels is now CLIA-waved. More data are needed on how these panels are used and interpreted in outpatient and urgent care settings.

\section{Next-Generation Sequencing and Clinical Metagenomics}

The combination of shotgun next-generation sequencing with metagenomic analyses of the data, directly from a clinical sample for the diagnosis of an infection is referred to as "clinical metagenomics". The ability to interrogate the sequencing data for any potential pathogen has resulted in tremendous promise for this methodologic approach. The first publication describing the real-time use of clinical metagenomics came in 2014 [61]. Since that time, several laboratories, including the laboratory who co-authored that initial case study, have validated metagenomic analyses of next-generation sequencing data directly from a variety of clinical sample types. A detailed description of next-generation sequencing and metagenomics methods is provided in the following references $(62,63)$. It is important to highlight that each laboratory has highly lab-and sample-type specific methods from sample preparation, library preparation, sequencing platform and depth, to bioinformatic analysis and interpretation. Any one of these components leads to some sort of bias, and there are currently no uniformly accepted criteria for any step in this process [64]. Thus, physicians who order this testing should understand, at least at a big-picture level, the associated limitations to adequately interpret results in the clinical context of their patients.

At the time of this writing, only one laboratory has validated metagenomic analyses of next-generation sequencing data from CSF. While this group has published several case reports over the last 5 years [65-68], they have recently published their prospective clinical study [69]. Some key takeaways from this larger sample set include the fact that unique detections by clinical metagenomics were equally likely to represent a clinically significant pathogen as they were to represent an incidental finding. For those that were deemed clinically significant, $6 \%$ of the total study population, roughly half would have influenced treatment as determined by a review panel consisting of clinical microbiologists and infectious disease physicians. All of these unique, clinically significant detections were confirmed by targeted PCR or bacterial/fungal ribosomal PCR followed by Sanger sequencing. This suggests that the true value of clinical metagenomics is in the unbiased nature of the method rather than improved sensitivity over targeted PCRs or ribosomal Sanger sequencing in CSF samples. The samples that were incidental detections included 10 detections of HIV, 7 of which were from patients known to be positive for HIV, HHV-7 $(n=3)$, HHV-6 $(n=2$, discussed above with the syndromic CSF panel), $\operatorname{EBV}(n=1)$, and 1 detection each of $\mathrm{HCV}$ and coronavirus. How these would be interpreted on an individual patient level in real time remains to be established. Equally informative are the infections not detected by their assay. In the supplemental material, the authors describe 11 cases diagnosed by serology and 7 cases diagnosed by an alternative sample type. However, there are also eight cases in which pathogen titers were considered too low to meet their cutoff for positivity, 6 of which had detectable reads below their clinically validated thresholds. This suggests that the current iteration of their test should be used in combination with conventional testing including targeted PCRs that may be more analytically sensitive for some pathogens such as Mycobacterium tuberculosis.

Again, at the time of this writing, only one laboratory has validated clinical metagenomics from plasma. Their test is based upon the hypothesis that, like placental or tumor DNA, pathogen DNA can be found in the bloodstream after breakdown and release from distal organs as well as from blood stream infections [70]. They call this "cell free" DNA based on the terminology used in human clinical genomics. There have been a few case reports showing utility for the diagnosis of culture-negative endocarditis and invasive Mycobacterium chimaera disease [71, 72]. In a single center clinical study looking at results of this test sent from children with a variety of indications, Rossoff et al. found a $70 \%$ positivity rate [73]. Sixty percent of these positives were also positive by conventional testing. For those that were only detected via clinical metagenomics, half were deemed clinically significant and half were considered insignificant, similar to the findings in the CSF study described above.

Other sources that have either been validated or are close to clinical validation for metagenomics include respiratory specimens $[64,74]$ and prosthetic joint specimens [75-77], respectively. Studies in these sample types also detect a number of questionable or insignificant positive results, highlighting the need for careful clinical interpretation. As this methodology may become mainstream in the future of clinical microbiology, appropriate utilization and interpretation will be key to cost-effectiveness.

\section{Additional Future Directions}

As the landscape of infectious diseases evolves, emerging resistance mechanisms and novel therapeutics for treatmentretractile diseases will require cutting edge molecular 
diagnostics to evolve simultaneously. The use of metagenomic and/or transcriptomic data to assess the resistome of a clinical sample requires further exploration to understand whether phenotypic antimicrobial susceptibility testing can ever be replaced, or at least sufficiently augmented, by such techniques. Tremendously promising work is also being done to understand how the microbiome influences human health and how that can be manipulated for treatment purposes for multi-drug-resistant organisms as well as $C$. difficile. The success of fecal microbiome transplants may someday require companion, real-time metagenomic characterization of donor stool as well as post-microbiome engraftment analyses [78]. Similarly, phage therapy, with or without CRISPR-Cas9 strategies, to combat highly antimicrobial resistant bacteria may require metagenomic analyses to assess efficacy [79, 80].

Finally, advances in the speed of amplification through techniques like "extreme PCR" will no doubt lead to a nextgeneration of rapid diagnostics [81]. Not mentioned in this review, due to scope, are systems like T2 Biosystems and Qvella, that test for bloodstream infections directly from a blood sample without the need for culture [82]. These types of technologies will no doubt continue to develop to reach the rapid state. Advances in molecular speed and cost will likely also result in the availability of direct access CLIA-waived molecular testing that patients can perform in their homes as well as a continuously expanding menu of molecular testing to be performed at the point-of-care, including the outpatient setting [83]. Hopefully, future iterations or new platforms will include smaller, modular, or even reflexive panels that lead to clinically appropriate testing for the right patient populations. Studies on the clinical utility, impact, and diagnostic and antimicrobial stewardship approaches for these types of advances are very much needed.

\section{Conclusions}

As molecular diagnostics continue to push the boundaries of speed and comprehensive and complex analyses, optimal utilization and impact will require partnership with stakeholders, particularly antimicrobial and diagnostic stewardship programs. The current pace of these technologic advances, and subsequent FDA approval of a subset, has created an unfortunate lag in our ability to appropriately steward their use. While many groups have published promising data on effective utilization we are far from the optimal state. More data is needed to drive the future use and expansion of these panels, particularly as they move closer to the point-of-care.

\section{Compliance with Ethical Standards}

Conflict of Interest Erin Graf declares that she has no conflict of interest. Preeti Pancholi declares that she has no conflict of interest.
Human and Animal Rights and Informed Consent This article does not contain any studies with human or animal subjects performed by any of the authors.

\section{References}

1. Emmadi R, Boonyaratanakornkit JB, Selvarangan R, Shyamala V, Zimmer BL, Williams L, et al. Molecular methods and platforms for infectious diseases testing a review of FDA-approved and cleared assays. J Mol Diagn. 2011;13(6):583-604.

2. Rosemary C. She JMB. Advances in Rapid Molecular Blood Culture Diagnostics: Healthcare Impact, Laboratory Implications, and Multiplex Technologies. J Appl Lab Med 2019;3(4):617-630

3. Banerjee R, Teng CB, Cunningham SA, Ihde SM, Steckelberg JM, Moriarty JP, et al. Randomized trial of rapid multiplex polymerase chain reaction-based blood culture identification and susceptibility testing. Clin Infect Dis. 2015;61(7):1071-80.

4. Bauer KA, West JE, Balada-Llasat JM, Pancholi P, Stevenson KB, Goff DA. An antimicrobial stewardship program's impact with rapid polymerase chain reaction methicillin-resistant Staphylococcus aureus/S. aureus blood culture test in patients with $\mathrm{S}$. aureus bacteremia. Clin Infect Dis. 2010;51(9):1074-80.

5. Frye AM, Baker CA, Rustvold DL, Heath KA, Hunt J, Leggett JE, et al. Clinical impact of a real-time PCR assay for rapid identification of staphylococcal bacteremia. J Clin Microbiol. 2012;50(1): 127-33.

6. Couturier MR, Bard JD. Direct-from-specimen pathogen identification: evolution of Syndromic panels. Clin Lab Med. 2019;39(3): 433-51.

7. Hanson KE, Couturier MR. Multiplexed molecular diagnostics for respiratory, gastrointestinal, and central nervous system infections. Clin Infect Dis. 2016;63(10):1361-7.

8. Ramanan P, Bryson AL, Binnicker MJ, Pritt BS, Patel R. Syndromic Panel-Based Testing in Clinical Microbiology. Clin Microbiol Rev. 2018;31(1): pii: e00024-17.

9. Messacar K, Parker SK, Todd JK, Dominguez SR. Implementation of rapid molecular infectious disease diagnostics: the role of diagnostic and antimicrobial stewardship. J Clin Microbiol. 2017;55(3): 715-23.

10. Lee BR, Hassan F, Jackson MA, Selvarangan R. Impact of multiplex molecular assay turn-around-time on antibiotic utilization and clinical management of hospitalized children with acute respiratory tract infections. J Clin Virol. 2019;110:11-6.

11. Rappo U, Schuetz AN, Jenkins SG, Calfee DP, Walsh TJ, Wells MT, et al. Impact of early detection of respiratory viruses by multiplex PCR assay on clinical outcomes in adult patients. J Clin Microbiol. 2016;54(8):2096-103.

12. Rogers BB, Shankar P, Jerris RC, Kotzbauer D, Anderson EJ, Watson JR, et al. Impact of a rapid respiratory panel test on patient outcomes. Arch Pathol Lab Med. 2015;139(5):636-41.

13. Sakata KK, Azadeh N, Brighton A, Temkit M, Klassen CL, Grys TE, et al. Impact of nasopharyngeal film array respiratory panel results on antimicrobial decisions in hospitalized patients. Can Respir J. 2018;2018:9821426.

14. Weiss ZF, Cunha CB, Chambers AB, Carr AV, Rochat C, RaglowDefranco M, et al. Opportunities Revealed for Antimicrobial Stewardship and Clinical Practice with Implementation of a Rapid Respiratory Multiplex Assay. J Clin Microbiol. 2019;57(10): pii: e00861-19.

15. Brendish NJ, Malachira AK, Armstrong L, Houghton R, Aitken S, Nyimbili E, et al. Routine molecular point-of-care testing for respiratory viruses in adults presenting to hospital with acute respiratory illness (ResPOC): a pragmatic, open-label, randomised controlled trial. Lancet Respir Med. 2017;5(5):401-11. 
16. Andrews D, Chetty Y, Cooper BS, Virk M, Glass SK, Letters A, et al. Multiplex PCR point of care testing versus routine, laboratorybased testing in the treatment of adults with respiratory tract infections: a quasi-randomised study assessing impact on length of stay and antimicrobial use. BMC Infect Dis. 2017;17(1):671.

17. Srinivas P, Rivard KR, Pallotta AM, Athans V, Martinez K, Loutzenheiser $\mathrm{S}$, et al. Implementation of a stewardship initiative on respiratory viral PCR-based antibiotic Deescalation. Pharmacotherapy. 2019;39(6):709-17.

18. Buss SN, Leber A, Chapin K, Fey PD, Bankowski MJ, Jones MK, et al. Multicenter evaluation of the BioFire FilmArray gastrointestinal panel for etiologic diagnosis of infectious gastroenteritis. $\mathrm{J}$ Clin Microbiol. 2015;53(3):915-25.

19. Khare R, Espy MJ, Cebelinski E, Boxrud D, Sloan LM, Cunningham SA, et al. Comparative evaluation of two commercial multiplex panels for detection of gastrointestinal pathogens by use of clinical stool specimens. J Clin Microbiol. 2014;52(10):3667-73.

20. Spina A, Kerr KG, Cormican M, Barbut F, Eigentler A, Zerva L, et al. Spectrum of enteropathogens detected by the FilmArray GI panel in a multicentre study of community-acquired gastroenteritis. Clin Microbiol Infect. 2015;21(8):719-28.

21. Valenzuela C, Legarraga P, Pena A, Arenas A, Berkowitz L, Ramirez G, et al. Etiologic and clinical characterization of community acquired gastroenteritis in adult patients in a Chilean emergency room by the FilmArray GI panel. PLoS One. 2018;13(11): e0207850.

22. Keske S, Zabun B, Aksoy K, Can F, Palaoglu E, Ergonul O. Rapid Molecular Detection of Gastrointestinal Pathogens and Its Role in Antimicrobial Stewardship. J Clin Microbiol. 2018;56(5): pii: e00148-18.

23. Kimon Clarke HT, Bridon I, Rogatcheva M, Demogines A, Henderson D, Saif D, et al. Vibrio cholerae Detection by the FilmArray ${ }^{\circledR}$ Gastrointestinal (GI) Panel. Open Forum Infectious Diseases. 2017;4(suppl_1):S605.

24. Mhaissen MN, Rodriguez A, Gu Z, Zhu H, Tang L, Sun Y, et al. Epidemiology of diarrheal illness in pediatric oncology patients. J Pediatric Infect Dis Soc. 2017;6(3):275-80.

25. Hitchcock MM, Gomez CA, Banaei N. Low Yield of FilmArray GI Panel in Hospitalized Patients with Diarrhea: an Opportunity for Diagnostic Stewardship Intervention. J Clin Microbiol. 2018;56(3): pii: e01558-17.

26. Shane AL, Mody RK, Crump JA, Tarr PI, Steiner TS, Kotloff K, et al. 2017 Infectious Diseases Society of America clinical Practice guidelines for the diagnosis and Management of Infectious Diarrhea. Clin Infect Dis. 2017;65(12):1963-73.

27. Schultz K, Sickbert-Bennett E, Marx A, Weber DJ, DiBiase LM, Campbell-Bright $\mathrm{S}$, et al. Preventable Patient Harm: a Multidisciplinary, Bundled Approach to Reducing Clostridium difficile Infections While Using a Glutamate Dehydrogenase/ Toxin Immunochromatographic Assay/Nucleic Acid Amplification Test Diagnostic Algorithm. J Clin Microbiol. 2018;56(9): pii:e00625-18.

28. Martin JS, Monaghan TM, Wilcox MH. Clostridium difficile infection: epidemiology, diagnosis and understanding transmission. Nat Rev Gastroenterol Hepatol. 2016;13(4):206-16.

29. Fang FC, Polage CR, Wilcox MH. Point-counterpoint: what is the optimal approach for detection of Clostridium difficile infection? J Clin Microbiol. 2017;55(3):670-80.

30. Truong CY, Gombar S, Wilson R, Sundararajan G, Tekic N, Holubar M, et al. Real-time electronic tracking of diarrheal episodes and laxative therapy enables verification of Clostridium difficile clinical testing criteria and reduction of Clostridium difficile infection rates. J Clin Microbiol. 2017;55(5):1276-84

31. Leber AL, Everhart K, Balada-Llasat JM, Cullison J, Daly J, Holt S, et al. Multicenter evaluation of BioFire FilmArray meningitis/ encephalitis panel for detection of Bacteria, viruses, and yeast in cerebrospinal fluid specimens. J Clin Microbiol. 2016;54(9):225161.

32. Schmitz JE, Tang YW. The GenMark ePlex((R)): another weapon in the syndromic arsenal for infection diagnosis. Future Microbiol. 2018;13:1697-708

33. Hanson KE, Slechta ES, Killpack JA, Heyrend C, Lunt T, Daly JA, et al. Preclinical assessment of a fully automated multiplex PCR panel for detection of central nervous system pathogens. J Clin Microbiol. 2016;54(3):785-7.

34. O'Halloran JA, Franklin A, Lainhart W, Burnham CA, Powderly W, Dubberke E. Pitfalls Associated With the Use of Molecular Diagnostic Panels in the Diagnosis of Cryptococcal Meningitis. Open Forum Infect Dis. 2017;4(4):ofx242.

35. Lewis PO, Lanier CG, Patel PD, Krolikowski WD, Krolikowski MA. False negative diagnostic errors with polymerase chain reaction for the detection of cryptococcal meningoencephalitis. Med Mycol. 2019: pii: myz064.

36. Slenker AK, Royer TL, Villalobos T. Human Herpesvirus 6 positivity on the FilmArray meningitis/encephalitis panel needs clinical interpretation. Clin Infect Dis. 2019;69(1):192-4.

37. Green DA, Pereira M, Miko B, Radmard S, Whittier S, Thakur K. Clinical significance of human Herpesvirus 6 positivity on the FilmArray meningitis/encephalitis panel. Clin Infect Dis. 2018;67(7):1125-8.

38. Olson CA, Dominguez SR, Miller S, Chiu CY, Messacar K. Gastroenteritis, hepatitis, encephalopathy, and human Herpesvirus 6 detection in an Immunocompetent child: benefits and risks of Syndromic multiplex molecular panel testing. J Pediatr. 2019;212: 228-31.

39. Gonzalez-Donapetry P, Garcia-Rodriguez J, Cendejas-Bueno E. A case of a FilmArray ((R)) ME false negative in meningococcal meningitis. J Inf Secur. 2019;79(3):277-87.

40. Graf EH, Farquharson MV, Cardenas AM. Comparative evaluation of the FilmArray meningitis/encephalitis molecular panel in a pediatric population. Diagn Microbiol Infect Dis. 2017;87(1):92-4.

41. Liesman RM, Strasburg AP, Heitman AK, Theel ES, Patel R, Binnicker MJ. Evaluation of a Commercial Multiplex Molecular Panel for Diagnosis of Infectious Meningitis and Encephalitis. J Clin Microbiol. 2018;56(4): pii: e01927-17.

42. Dien Bard J, Alby K. Point-Counterpoint: Meningitis/Encephalitis Syndromic Testing in the Clinical Laboratory. J Clin Microbiol. 2018;56(4): pii: e00018-18.

43. Radmard S, Reid S, Ciryam P, Boubour A, Ho N, Zucker J, et al. Clinical utilization of the FilmArray meningitis/encephalitis (ME) multiplex polymerase chain reaction (PCR) assay. Front Neurol. 2019;10:281.

44. Nabower AM, Miller S, Biewen B, Lyden E, Goodrich N, Miller A, et al. Association of the FilmArray meningitis/encephalitis panel with clinical management. Hosp Pediatr. 2019;9(10):763-9.

45. DiDiodato G, Bradbury N. Cerebrospinal Fluid Analysis With the BioFire FilmArray Meningitis/Encephalitis Molecular Panel Reduces Length of Hospital Stay in Patients With Suspected Central Nervous System Infections. Open Forum Infect Dis. 2019;6(4):ofz119.

46. Dack K, Pankow S, Ablah E, Zackula R, Assi M. Contribution of the BioFire $((\mathrm{R}))$ FilmArray $((\mathrm{R}))$ meningitis/encephalitis panel: assessing antimicrobial duration and length of stay. Kans J Med. 2019;12(1):1-3.

47. Wilen CB, Monaco CL, Hoppe-Bauer J, Jackups R Jr, Bucelli RC, Burnham CA. Criteria for reducing unnecessary testing for herpes simplex virus, varicella-zoster virus, cytomegalovirus, and enterovirus in cerebrospinal fluid samples from adults. J Clin Microbiol. 2015;53(3):887-95.

48. Messacar K, Robinson CC, Dominguez SR. Letter to the editor: economic analysis lacks external validity to support universal 
syndromic testing for suspected meningitis/encephalitis. Future Microbiol. 2018;13:1553-4.

49. Levitz RE. Herpes simplex encephalitis: a review. Heart Lung. 1998;27(3):209-12.

50. Pinsky BA, Hayden RT. Cost-Effective Respiratory Virus Testing. J Clin Microbiol. 2019;57(9): pii: e00373-19.

51. Azar MM, Landry ML. Detection of Influenza A and B Viruses and Respiratory Syncytial Virus by Use of Clinical Laboratory Improvement Amendments of 1988 (CLIA)-Waived Point-ofCare Assays: a Paradigm Shift to Molecular Tests. J Clin Microbiol. 2018;56(7): e00367-18.

52. Pritt BS, Patel R, Kirn TJ, Thomson RB Jr. Point-counterpoint: a nucleic acid amplification test for Streptococcus pyogenes should replace antigen detection and culture for detection of bacterial pharyngitis. J Clin Microbiol. 2016;54(10):2413-9.

53. Garvey MI, Wilkinson MAC, Bradley CW, Biggs M, ReddyKolanu V, Osman H, et al. Impact of a PCR point of care test for influenza $\mathrm{a} / \mathrm{B}$ on an acute medical unit in a large UK teaching hospital: results of an observational, pre and post intervention study. Antimicrob Resist Infect Control. 2019;8:120.

54. Benirschke RC, McElvania E, Thomson RB, Jr., Kaul KL, Das S. Clinical Impact of Rapid Point-of-Care PCR Influenza Testing in an Urgent Care Setting: a Single-Center Study. J Clin Microbiol. 2019;57(3): pii: e01281-18.

55. Martinot M, Greigert V, Gravier S, Klein S, Eyriey M, Pachart A, et al. Positive Impact of a Point-of-Care Molecular Influenza Test in the Emergency Department During the 2017-2018 Seasonal Influenza Epidemic. Open Forum Infect Dis 2019;6(7): ofz312.

56. Rao A, Berg B, Quezada T, Fader R, Walker K, Tang S, et al. Diagnosis and antibiotic treatment of group a streptococcal pharyngitis in children in a primary care setting: impact of point-of-care polymerase chain reaction. BMC Pediatr. 2019;19(1):24.

57. Guy RJ, Ward J, Causer LM, Natoli L, Badman SG, Tangey A, et al. Molecular point-of-care testing for chlamydia and gonorrhoea in indigenous Australians attending remote primary health services (TTANGO): a cluster-randomised, controlled, crossover trial. Lancet Infect Dis. 2018;18(10):1117-26.

58. Keizur EM, Goldbeck C, Vavala G, Romero A, Ocasio M, Fournier $\mathrm{J}$, et al. Safety and effectiveness of same-day chlamydia trachomatis and Neisseria gonorrhoeae screening and treatment among gay, Bisexual, Transgender, and Homeless Youth in Los Angeles, California and New Orleans, Louisiana. Sex Transm Dis. 2019;47(1):19-23.

59. K. L. New Requirements for Molecular Micro Waived Testing. CAP TODAY. 2019;:September.

60. Donato LJ, Myhre NK, Murray MA, McDonah MR, Myers JF, Maxson JA, et al. Assessment of Test Performance and Potential for Environmental Contamination Associated with a Point-of-Care Molecular Assay for Group A Streptococcus in an End User Setting. J Clin Microbiol. 2019;57(2): e01629-18.

61. Wilson MR, Naccache SN, Samayoa E, Biagtan M, Bashir H, Yu G, et al. Actionable diagnosis of neuroleptospirosis by next-generation sequencing. N Engl J Med. 2014;370(25):2408-17.

62. Chiu CY, Miller SA. Clinical metagenomics. Nat Rev Genet. 2019;20(6):341-55.

63. Quince C, Walker AW, Simpson JT, Loman NJ, Segata N. Shotgun metagenomics, from sampling to analysis. Nat Biotechnol. 2017;35(9):833-44.

64. Schlaberg R, Chiu CY, Miller S, Procop GW, Weinstock G, Professional Practice $\mathrm{C}$, et al. Validation of metagenomic nextgeneration sequencing tests for universal pathogen detection. Arch Pathol Lab Med. 2017;141(6):776-86.
65. Chiu CY, Coffey LL, Murkey J, Symmes K, Sample HA, Wilson MR, et al. Diagnosis of fatal human case of St. Louis encephalitis virus infection by metagenomic sequencing, California, 2016. Emerg Infect Dis. 2017;23(10):1964-8.

66. Greninger AL, Messacar K, Dunnebacke T, Naccache SN, Federman S, Bouquet J, et al. Clinical metagenomic identification of Balamuthia mandrillaris encephalitis and assembly of the draft genome: the continuing case for reference genome sequencing. Genome Med. 2015;7:113.

67. Mongkolrattanothai K, Naccache SN, Bender JM, Samayoa E, Pham E, Yu G, et al. Neurobrucellosis: unexpected answer from metagenomic next-generation sequencing. J Pediatric Infect Dis Soc. 2017;6(4):393-8.

68. Naccache SN, Peggs KS, Mattes FM, Phadke R, Garson JA, Grant $\mathrm{P}$, et al. Diagnosis of neuroinvasive astrovirus infection in an immunocompromised adult with encephalitis by unbiased nextgeneration sequencing. Clin Infect Dis. 2015;60(6):919-23.

69. Wilson MR, Sample HA, Zorn KC, Arevalo S, Yu G, Neuhaus J, et al. Clinical metagenomic sequencing for diagnosis of meningitis and encephalitis. N Engl J Med. 2019;380(24):2327-40.

70. Blauwkamp TA, Thair S, Rosen MJ, Blair L, Lindner MS, Vilfan ID, et al. Analytical and clinical validation of a microbial cell-free DNA sequencing test for infectious disease. Nat Microbiol. 2019;4(4):663-74.

71. Kondo M, Dalai SC, Venkatasubrahmanyam S, Eisenberg N, Robinson BD, Westblade LF, et al. Diagnosis and Genotyping of Coxiella burnetii Endocarditis in a Patient with Prosthetic Pulmonary Valve Replacement Using Next-Generation Sequencing of Plasma Microbial Cell-Free DNA. Open Forum Infect Dis. 2019;6(6):ofz242.

72. Nomura J, Rieg G, Bluestone G, Tsai T, Lai A, Terashita D, et al. Rapid detection of invasive Mycobacterium chimaera disease via a novel plasma-based next-generation sequencing test. BMC Infect Dis. 2019;19(1):371.

73. Rossoff J, Chaudhury S, Soneji M, Patel SJ, Kwon S, Armstrong A, et al. Noninvasive Diagnosis of Infection Using Plasma NextGeneration Sequencing: A Single-Center Experience. Open Forum Infect Dis. 2019;6(8): pii: ofz327.

74. Schlaberg R, Queen K, Simmon K, Tardif K, Stockmann C, Flygare $\mathrm{S}$, et al. Viral pathogen detection by Metagenomics and pan-viral group polymerase chain reaction in children with pneumonia lacking identifiable etiology. J Infect Dis. 2017;215(9):1407-15.

75. Ivy MI, Thoendel MJ, Jeraldo PR, Greenwood-Quaintance KE, Hanssen AD, Abdel MP, et al. Direct Detection and Identification of Prosthetic Joint Infection Pathogens in Synovial Fluid by Metagenomic Shotgun Sequencing. J Clin Microbiol. 2018;56(9): pii: e00402-18.

76. Thoendel M, Jeraldo P, Greenwood-Quaintance KE, Chia N, Abdel MP, Steckelberg JM, et al. A novel prosthetic joint infection pathogen, mycoplasma salivarium, identified by metagenomic shotgun sequencing. Clin Infect Dis. 2017;65(2):332-5.

77. Thoendel MJ, Jeraldo PR, Greenwood-Quaintance KE, Yao JZ, Chia N, Hanssen AD, et al. Identification of prosthetic joint infection pathogens using a shotgun Metagenomics approach. Clin Infect Dis. 2018;67(9):1333-8.

78. Allegretti JR, Mullish BH, Kelly C, Fischer M. The evolution of the use of faecal microbiota transplantation and emerging therapeutic indications. Lancet. 2019;394(10196):420-31.

79. Strich JR, Chertow DS. CRISPR-Cas Biology and Its Application to Infectious Diseases. J Clin Microbiol. 2019;57(4): pii: e0130718. 
80. Tse BN, Adalja AA, Houchens C, Larsen J, Inglesby TV, Hatchett R. Challenges and opportunities of nontraditional approaches to treating bacterial infections. Clin Infect Dis. 2017;65(3):495-500.

81. Millington AL, Houskeeper JA, Quackenbush JF, Trauba JM, Wittwer CT. The kinetic requirements of extreme qPCR. Biomol Detect Quantif. 2019;17:100081.

82. Nguyen MH, Clancy CJ, Pasculle AW, Pappas PG, Alangaden G, Pankey GA, et al. Performance of the T2Bacteria panel for diagnosing bloodstream infections: a diagnostic accuracy study. Ann Intern Med. 2019;170(12):845-52.

83. Kozel TR, Burnham-Marusich AR. Point-of-care testing for infectious diseases: past, present, and future. J Clin Microbiol. 2017;55(8):2313-20.

Publisher's Note Springer Nature remains neutral with regard to jurisdictional claims in published maps and institutional affiliations. 